\title{
The Bleeding Risk after Endoscopic Ultrasound-Guided Fine Needle Aspiration in Patients Taking Antithrombotic Agents Is Not Negligible
}

\author{
Dong Kee Jang ${ }^{1}$ and Sang Hyub Lee ${ }^{2}$ \\ ${ }^{1}$ Department of Internal Medicine, Dongguk University Ilsan Hospital, Goyang, and ${ }^{2}$ Department of Internal Medicine and Liver Research \\ Institute, Seoul National University Hospital, Seoul National University College of Medicine, Seoul, Korea
}

See "A Prospective Multicenter Study Evaluating Bleeding Risk after Endoscopic Ultrasound-Guided Fine Needle Aspiration in Patients Prescribed Antithrombotic Agents" by Kazumichi Kawakubo, et al. on page 353, Vol. 12. No. 3, 2018

Endoscopic ultrasound-guided fine needle aspiration (EUSFNA) is the established standard method for the histological diagnosis of various gastrointestinal malignancies and peri-intestinal structures. ${ }^{1}$ Peri-intestinal structures include lymph nodes, masses in the pancreas, liver, adrenal gland, and bile duct that are accessible from the gastrointestinal tract. EUS-FNA is indispensable especially in the diagnosis of pancreatic malignancies. Moreover, as the procedure has evolved, it has become possible to perform various magic interventions by modifying EUS-FNA. Therefore, the applications of EUS including EUS-FNA are getting much more attention worldwide. The more often it is performed, the more attention is needed to its complications.

Bleeding is one of the significant adverse events after EUSFNA. The overall adverse event associated with EUS-FNA was reported to be $0.98 \%$ including a bleeding rate of $0.13 \%$ $(14 / 10,941)$ according to a recent meta-analysis. ${ }^{2}$ EUS-FNA is defined as a high-risk procedure in the guidelines of the European and American Society of Gastrointestinal Endoscopy.,4 However, the basis of these guidelines is relatively weak since most relevant studies were retrospective, and there were few studies on the risk of bleeding in patients taking antithrombotic agents. Kawakubo et al., ${ }^{5}$ prospectively evaluated the bleeding risk of EUS-FNA in patients taking antithrombotic agents. This study is of great significance in that there have been few studies of this kind in the past and that it was a prospective multicenter study in Japan. In the study, bleeding after EUS-FNA occurred in two of 85 (2.4\%; 95\% CI, 0.6\% to $8.3 \%)$ patients taking antithrombotic agents. One was receiving dual antiplatelet therapy and the other was taking warfarin. Both of them underwent bleeding in spite of discontinuation of thienopyridine and warfarin in advance, respectively. The authors concluded that the rate of bleeding after EUS-FNA in patients taking antithrombotic agents might be considerable. Although this study was conducted on a small number of patients with limited statistical power, which may well affect EUS-FNA practice in the future because there were few similar prospective studies in the past.

A recent retrospective study on the similar subject reported that the overall bleeding rate was $0.9 \%(7 / 742){ }^{6}$ In detail, 131 patients $(17.7 \%)$ were on antithrombotic therapy in 742 patients and six of seven bleeding occurred in patients who did not take antithrombotic agents. Bleeding occurred in one patient (1.6\%) among the 61 patients who maintained use of aspirin or cilostazol, and no bleeding was detected in 62 patients who had discontinued antithrombotic agents. Based on these results, the authors concluded that the bleeding rate was low even in patients who underwent EUS-FNA while continuing aspirin or cilostazol. However, this study was a retrospective study with a small number of patients. So, it is difficult to generalize the results. There has been a similar study in the past. Kien-Fong $\mathrm{Vu}$ et al., ${ }^{7}$ observed no bleeding events in 26 patients taking aspirin or nonsteroidal anti-inflammatory drugs, while seven of 190 bleeding events (3.7\%) occurred in control groups. Since the study also included a small number of patients and guidelines were not established at that time, it is also difficult to generalize at present.

The American and European guidelines recommend that aspirin should not be discontinued prior to EUS-FNA regardless of thrombotic risk, but thienopyridine and anticoagulants should

Correspondence to: Sang Hyub Lee

Department of Internal Medicine and Liver Research Institute, Seoul National University Hospital, Seoul National University College of Medicine, 101 Daehak-ro, Jongno-gu, Seoul 03080, Korea

Tel: +82-2-2072-4892, Fax: +82-2-762-9662, E-mail: gidoctor@snuh.org

pISSN 1976-2283 eISSN 2005-1212 https://doi.org/10.5009/gnl18103

(c) This is an Open Access article distributed under the terms of the Creative Commons Attribution Non-Commercial License (http://creativecommons.org/licenses/by-nc/4.0) which permits unrestricted non-commercial use, distribution, and reproduction in any medium, provided the original work is properly cited. 
be discontinued before the procedure. ${ }^{3,4}$ However, Kawakubo et al., ${ }^{5}$ reported that bleeding could occur even if antithromobitic agents were stopped in advance according to these guidelines. Though these drugs are stopped, the risk of bleeding does not seem to be lowered than expected. In summary, when EUS-FNA is performed in patients taking antithrombotic agents, thienopyridine and anticoagulants should be stopped in advance. Even if these drugs are stopped, care should be taken in consideration of the risk of bleeding during or after EUS-FNA.

\section{CONFLICTS OF INTEREST}

No potential conflict of interest relevant to this article was reported.

\section{REFERENCES}

1. Dumonceau JM, Deprez PH, Jenssen C, et al. Indications, results, and clinical impact of endoscopic ultrasound (EUS)-guided sampling in gastroenterology: European Society of Gastrointestinal Endoscopy (ESGE) clinical guideline - updated January 2017. Endoscopy 2017;49:695-714.

2. Wang KX, Ben QW, Jin ZD, et al. Assessment of morbidity and mortality associated with EUS-guided FNA: a systematic review. Gastrointest Endosc 2011;73:283-290.

3. Veitch AM, Vanbiervliet G, Gershlick AH, et al. Endoscopy in patients on antiplatelet or anticoagulant therapy, including direct oral anticoagulants: British Society of Gastroenterology (BSG) and European Society of Gastrointestinal Endoscopy (ESGE) guidelines. Endoscopy 2016;48:385-402.

4. ASGE Standards of Practice Committee, Acosta RD, Abraham NS, et al. The management of antithrombotic agents for patients undergoing GI endoscopy. Gastrointest Endosc 2016;83:3-16.

5. Kawakubo K, Yane K, Eto K, et al. A prospective multicenter study evaluating bleeding risk after endoscopic ultrasound-guided fine needle aspiration in patients prescribed antithrombotic agents. Gut Liver 2018;12:353-359.

6. Inoue T, Okumura F, Sano H, et al. Bleeding risk of endoscopic ultrasound-guided fine-needle aspiration in patients undergoing antithrombotic therapy. Dig Endosc 2017;29:91-96.

7. Kien-Fong Vu C, Chang F, Doig L, Meenan J. A prospective control study of the safety and cellular yield of EUS-guided FNA or Trucut biopsy in patients taking aspirin, nonsteroidal anti-inflammatory drugs, or prophylactic low molecular weight heparin. Gastrointest Endosc 2006;63:808-813. 